\title{
Gravitational instability of polytropic spheres and generalized thermodynamics
}

\author{
P. H. Chavanis ${ }^{\star}$ \\ Laboratoire de Physique Quantique, Université Paul Sabatier, 118 route de Narbonne 31062 Toulouse, France \\ Institute for Theoretical Physics, University of California, Santa Barbara, California CA93106, USA
}

Received 18 September 2001 / Accepted 30 January 2002

\begin{abstract}
We extend the existing literature on the structure and stability of polytropic gas spheres reported in the classical monograph of Chandrasekhar (1932). For isolated polytropes with index $1<n<5$, we provide a new, alternative, proof that the onset of gravitational instability occurs for $n=3$ and we express the perturbation profiles of density and velocity at the point of marginal stability in terms of the Milne variables. Then, we consider the case of polytropes confined within a box of radius $R$ (an extension of the Antonov problem for isothermal gas spheres). For $n \geq 3$, the mass-density relation presents damped oscillations and there exists a limiting mass above which no hydrostatic equilibrium is possible. As for isothermal gas spheres, the onset of instability occurs precisely at the point of maximum mass in the series of equilibrium. Analytical results are obtained for the particular index $n=5$. We also discuss the relation of our study with generalized thermodynamics (Tsallis entropy) recently investigated by Taruya \& Sakagami (2002).
\end{abstract}

Key words. hydrodynamics - instabilities - stars: oscillations

\section{Introduction}

In earlier papers of this series (Chavanis 2002a,b,c) we investigated the gravitational instability of finite isothermal spheres in Newtonian gravity and general relativity for classical particles and for quantum particles (fermions). From a theoretical point of view, these systems exhibit interesting behavior with the occurence of phase transitions associated with gravitational collapse (Antonov 1962; Lynden-Bell \& Wood 1968; Padmanabhan 1990). A rich stability analysis follows and can be conducted analytically or by using graphical constructions. From an astrophysical point of view, these studies can be relevant for various systems, including elliptical galaxies, globular clusters, the interstellar medium, the core of neutron stars and dark matter made of massive neutrinos.

In this paper, we propose to extend our study to the case of polytropic gas spheres. Polytropes with index $1<n<5$ are self-confined, so it is not necessary to introduce an artificial "box" to limit their spatial extent. Using the methods developed for isothermal configurations, we show that the transition from stability to instability corresponds to an index $n=3$. This result is well-known but we provide a new derivation based on the exact resolution of the pulsation equation for polytropes. The perturbation profiles at the point of marginal stability are

* e-mail: chavanis@irsamc2.ups-tlse.fr expressed in terms of the Milne variables (Chandrasekhar 1932). The profile of density perturbation has only one node and the velocity perturbation is proportional to the radial distance.

Then, we consider the case of polytropes with arbitrary index $n>1$ confined within a box of radius $R$. For $n=\infty$, we recover the classical Antonov (1962) problem for isothermal gas spheres. However, for $n \geq 3$ we already obtain results strikingly similar to those obtained for isothermal configurations. In particular, confined polytropes in hydrostatic equilibrium can exist only below a limiting mass (for a given box radius $R$ ) and the series of equilibrium becomes unstable precisely at the point of maximum mass. Subsequent oscillations in the massdensity profile (for $n>5$ ) are associated with secondary modes of instability. The locii of these modes of instability follow a geometric progression with a ratio depending on the index of the polytrope. For $n=\infty$, we recover the ratio $10.74 \ldots$ of isothermal gas spheres.

While this paper was in preparation, we came across the preprint of Taruya \& Sakagami (2002) on a related subject. These authors investigate the stability of polytropes in the framework of extended thermodynamics, using Tsallis entropy. Therefore, in Sect. 4, we analyze the connexion of the present study with their approach and we discuss the relevance of Tsallis entropy for describing astrophysical systems. 


\section{Properties of polytropic gas spheres}

\subsection{The Lane-Emden equation}

Polytropic stars are characterized by an equation of state of the form

$p=K \rho^{\gamma}$,

where $K$ and $\gamma$ are constants. The index $n$ of the polytrope is defined by the relation

$\gamma=1+\frac{1}{n}$

The equation of state (1) corresponds to an adiabatic equilibrium in regions where convection keeps the star stirred up and produces a uniform entropy distribution ( $s=$ const.). In that case, $\gamma$ is the ratio of specific heats $c_{p} / c_{V}$ at constant pressure and volume. For a monoatomic gas, $\gamma=5 / 3$. The equation of state (1) also describes a polytropic equilibrium characterized by a uniform specific heat $c \equiv \delta Q / \mathrm{d} T$. In this more general situation $\gamma=\left(c_{\mathrm{p}}-c\right) /\left(c_{V}-c\right)$. Convective equilibrium is recovered for $c=0$ and isothermal equilibrium is obtained in the limit of infinite specific heat $c \rightarrow+\infty$. A power law relation between the pressure and the density is also the limiting form of the equation of state describing a gas of cold degenerate fermions (Chandrasekhar 1932). In that case, the constant $K$ can be expressed in terms of fundamental constants. In the classical limit $\gamma=5 / 3$, $n=3 / 2$ and $K=\frac{1}{20}(3 / \pi)^{2 / 3} h^{2} / m$ (where $h$ is the Planck constant) and in the relativistic limit $\gamma=4 / 3, n=3$ and $K=\frac{1}{8}(3 / \pi)^{1 / 3} h c$ (where $c$ is the speed of light). Historically, the index $\gamma=5 / 3$ appears in the classical theory of white dwarf stars initiated by Fowler (1926) and the index $\gamma=4 / 3$ is related to the limiting mass of Chandrasekhar (1931).

The condition of hydrostatic equilibrium for a spherically symmetrical distribution of matter reads

$\frac{\mathrm{d} p}{\mathrm{~d} r}=-\rho \frac{\mathrm{d} \Phi}{\mathrm{d} r}$,

where $\Phi$ is the gravitational potential. Using the Gauss theorem

$\frac{\mathrm{d} \Phi}{\mathrm{d} r}=\frac{G M(r)}{r^{2}}$,

where $M(r)=\int_{0}^{r} \rho 4 \pi r^{2} \mathrm{~d} r$ is the mass contained within the sphere of radius $r$, we can derive the fundamental equation of equilibrium (Chandrasekhar 1932)

$\frac{1}{r^{2}} \frac{\mathrm{d}}{\mathrm{d} r}\left(\frac{r^{2}}{\rho} \frac{\mathrm{d} p}{\mathrm{~d} r}\right)=-4 \pi G \rho$.

Equations (1), (5) fully determine the structure of polytropic gas spheres. Letting

$\rho=\rho_{0} \theta^{n}, \quad \xi=\left[\frac{4 \pi G \rho_{0}^{1-1 / n}}{K(n+1)}\right]^{1 / 2} r$, where $\rho_{0}$ is the central density, we can reduce the condition of hydrostatic equilibrium to the Lane-Emden equation (Chandrasekhar 1932)

$\frac{1}{\xi^{2}} \frac{\mathrm{d}}{\mathrm{d} \xi}\left(\xi^{2} \frac{\mathrm{d} \theta}{\mathrm{d} \xi}\right)=-\theta^{n}$,

with boundary conditions

$\theta(0)=1, \quad \theta^{\prime}(0)=0$.

For $n>3$, the Lane-Emden equation admits an analytical solution which is singular at the origin:

$\theta_{s}=\left[\frac{2(n-3)}{(n-1)^{2}}\right]^{\frac{1}{n-1}} \frac{1}{\xi^{\frac{2}{n-1}}}$.

Regular solutions of the Lane-Emden equation must in general be computed numerically. For $\xi \rightarrow 0$, we can expand the function $\theta$ in Taylor series. The first terms in this expansion are given by

$\theta=1-\frac{1}{6} \xi^{2}+\frac{n}{120} \xi^{4}+\ldots$

The behavior of $\theta(\xi)$ at large distances deserves a more specific discussion. For $1<n<5$, the density falls off to zero at a finite radius $R$, identified as the radius of the star. If we denote by $\xi_{1}$ the value of the normalized distance at which $\theta=0$ then, for $\xi \rightarrow \xi_{1}$, we have

$\theta=-\xi_{1} \theta_{1}^{\prime}\left[\frac{\xi_{1}-\xi}{\xi_{1}}+\left(\frac{\xi_{1}-\xi}{\xi_{1}}\right)^{2}+\left(\frac{\xi_{1}-\xi}{\xi_{1}}\right)^{3}+\ldots\right]$.

For $n>5$, the polytropes extend to infinity, like the isothermal configurations recovered in the limit $n=\infty$. For $\xi \rightarrow+\infty$,

$$
\begin{aligned}
\theta=\left[\frac{2(n-3)}{(n-1)^{2}}\right]^{\frac{1}{n-1}} & \frac{1}{\xi^{\frac{2}{n-1}}}\left\{1+\frac{C}{\xi^{\frac{n-5}{2(n-1)}}}\right. \\
& \left.\times \cos \left[\frac{\sqrt{7 n^{2}-22 n-1}}{2(n-1)} \ln \xi+\delta\right]\right\} .
\end{aligned}
$$

The curve (12) intersects the singular solution (9) infinitely often at points that asymptotically increase geometrically in the ratio $1: \exp \left\{2(n-1) \pi / \sqrt{7 n^{2}-22 n-1}\right\}$. Since $\theta^{n} \sim \xi^{\frac{-2 n}{n-1}}$ at large distances, these configurations have an "infinite mass", which is clearly unphysical. In the following, we shall restrict these configurations to a "box" of radius $R$, as in the classical Antonov problem. Therefore, Eq. (7) must be solved for $\xi \leq \alpha$ with

$\alpha=\left[\frac{4 \pi G \rho_{0}^{1-1 / n}}{K(n+1)}\right]^{1 / 2} R$.

Note that, for a fixed box radius $R, \alpha$ is a measure of the central density $\rho_{0}$. The case $n=5$ is special. For this index, the Lane-Emden equation can be solved analytically and yields the result:

$\theta=\frac{1}{\left(1+\frac{1}{3} \xi^{2}\right)^{1 / 2}}$

The total mass of this configuration is finite but its potential energy diverges. Therefore, this polytrope must also be confined within a box. In Fig. 1, we have plotted different density profiles with index $n=3,5,6$. 


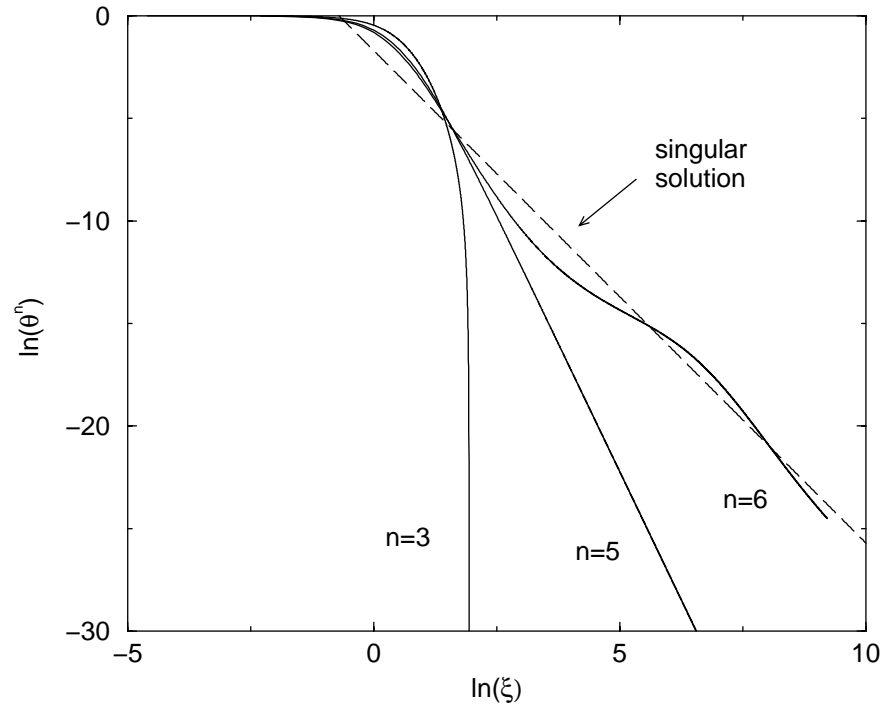

Fig. 1. Density profiles of polytropes with index $n=3,5,6$. The dashed line corresponds to the singular solution (9).

\subsection{The Milne variables}

As in the analysis of isothermal gas spheres, it will be convenient in the following to introduce the Milne variables $(u, v)$ defined by (Chandrasekhar 1932):

$u=-\frac{\xi \theta^{n}}{\theta^{\prime}}, \quad v=-\frac{\xi \theta^{\prime}}{\theta}$.

Taking the logarithmic derivative of $u$ and $v$ with respect to $\xi$ and using Eq. (7), we get

$$
\begin{aligned}
& \frac{1}{u} \frac{\mathrm{d} u}{\mathrm{~d} \xi}=\frac{1}{\xi}(3-n v-u), \\
& \frac{1}{v} \frac{\mathrm{d} v}{\mathrm{~d} \xi}=\frac{1}{\xi}(u+v-1) .
\end{aligned}
$$

Due to the homology invariance of the polytropic configuations (see Chandrasekhar 1932), the Milne variables $u$ and $v$ satisfy a first order differential equation

$\frac{u}{v} \frac{\mathrm{d} v}{\mathrm{~d} u}=-\frac{u+v-1}{u+n v-3}$

The solution curve in the $(u, v)$ plane (see Figs. 2-4) is parametrized by $\xi$. It starts from the point $(u, v)=(3,0)$ with a slope $(\mathrm{d} v / \mathrm{d} u)_{0}=-\frac{5}{3 n}$ as $\xi \rightarrow 0$. For $1<n<5$, the curve is monotonous and tends to $(u, v)=(0,+\infty)$ as $\xi \rightarrow \xi_{1}$. More precisely, using Eq. (11), we have

$$
u v^{n} \sim \omega_{n}^{n-1}, \quad \omega_{n}=-\xi_{1}^{\frac{n+1}{n-1}} \theta_{1}^{\prime} \quad\left(\xi \rightarrow \xi_{1}\right) .
$$

For $n>5$, the solution curve spirals indefinitely around the fixed point $\left(u_{\mathrm{s}}, v_{\mathrm{s}}\right)=\left(\frac{n-3}{n-1}, \frac{2}{n-1}\right)$, corresponding to the singular solution (9), as $\xi$ tends to infinity. All polytropic spheres must necessarily lie on this curve. For bounded polytropic spheres, $\xi$ must be terminated at the box radius $\alpha$. For $n=5$, the Milne variables are related according to $u+3 v=3$.

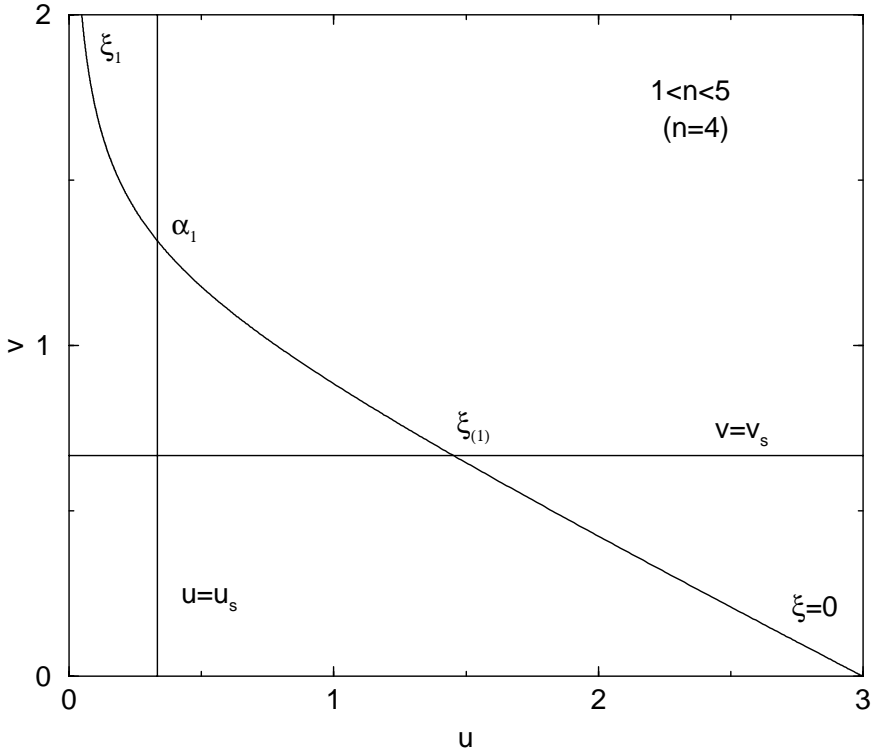

Fig. 2. The $(u, v)$ plane for polytropes with index $1<n<5$. The construction is made explicitly for $n=4$.

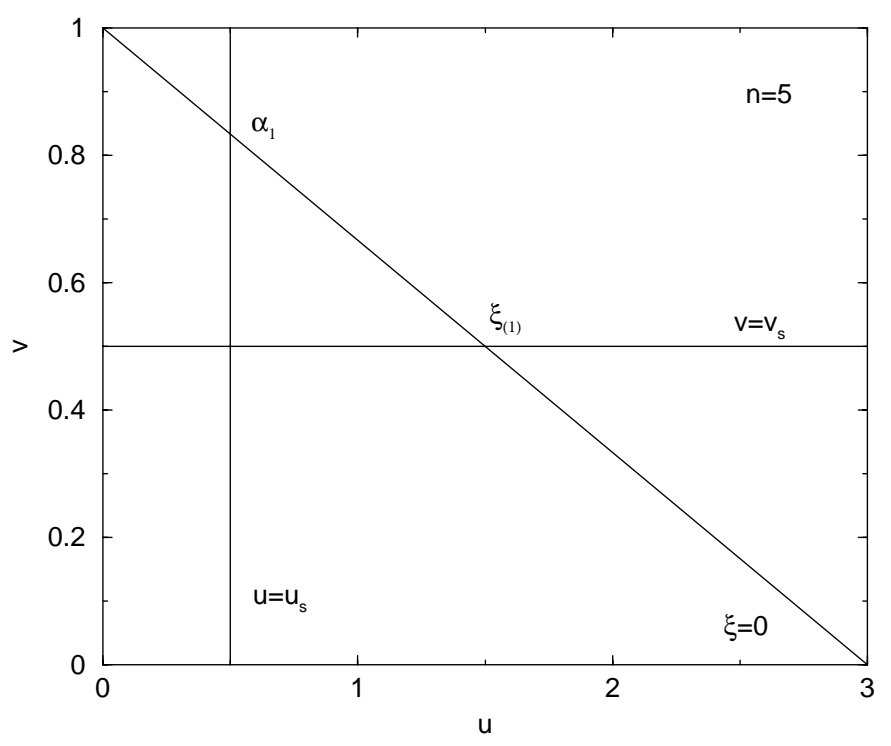

Fig. 3. The $(u, v)$ plane for polytropes with index $n=5$.

\subsection{The maximum mass and minimum temperature of confined polytropes}

For polytropes confined within a box of radius $R$, there exists a well-defined relation between the mass $M$ of the configuration and the central density $\rho_{0}$ (through the parameter $\alpha$ ). Starting from the relation

$$
\begin{aligned}
M & =\int_{0}^{R} \rho 4 \pi r^{2} \mathrm{~d} r \\
& =4 \pi \rho_{0}\left[\frac{K(1+n) \rho_{0}^{1 / n-1}}{4 \pi G}\right]^{3 / 2} \int_{0}^{\alpha} \theta^{n} \xi^{2} \mathrm{~d} \xi
\end{aligned}
$$

and using the Lane-Emden Eq. (7), we get

$$
M=-4 \pi \rho_{0}\left[\frac{K(1+n) \rho_{0}^{1 / n-1}}{4 \pi G}\right]^{3 / 2} \alpha^{2} \theta^{\prime}(\alpha) .
$$




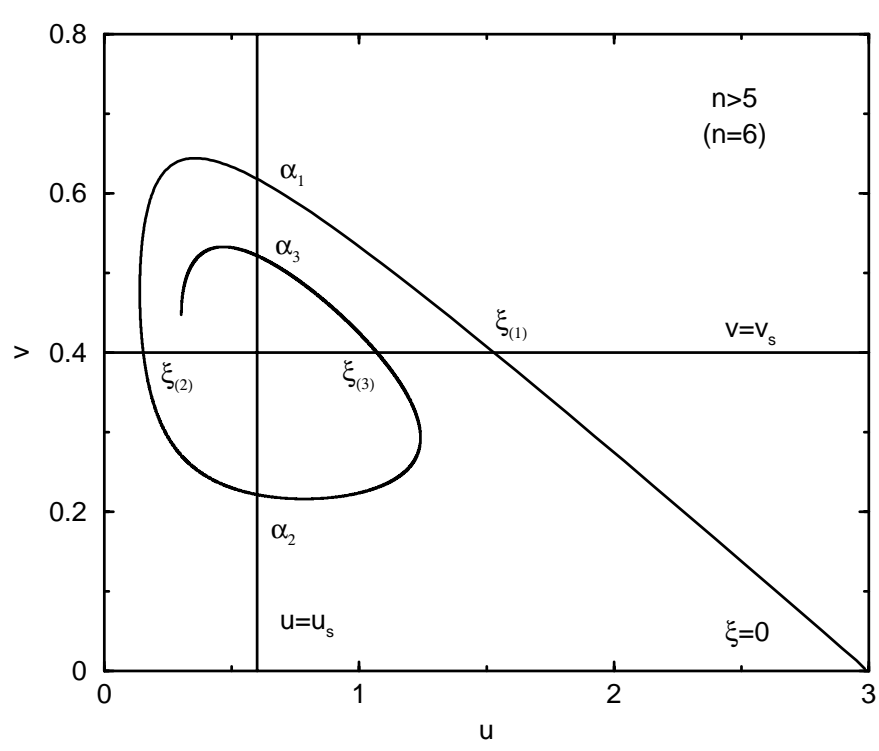

Fig. 4. The $(u, v)$ plane for polytropes with index $n>5$. The construction is made explicitly for $n=6$.

Expressing the central density in terms of $\alpha$, using Eq. (13), we obtain after some rearrangements

$M=-4 \pi\left[\frac{K(1+n)}{4 \pi G}\right]^{\frac{n}{n-1}} R^{\frac{n-3}{n-1}} \alpha^{\frac{n+1}{n-1}} \theta^{\prime}(\alpha)$.

Introducing the parameter

$\eta \equiv \frac{M}{4 \pi}\left[\frac{4 \pi G}{K(1+n)}\right]^{\frac{n}{n-1}} \frac{1}{R^{\frac{n-3}{n-1}}}$,

the foregoing relation can be rewritten

$\eta=-\alpha^{\frac{n+1}{n-1}} \theta^{\prime}(\alpha)$.

For $n<5$, the normalized box radius $\alpha$ in necessarily restricted by the inequality $\alpha \leq \xi_{1}$. For the limiting value $\alpha=\xi_{1}$, corresponding to an isolated polytrope satisfying $\rho(R)=0$, we have

$\eta\left(\xi_{1}\right)=\omega_{n}$.

The quantity $\omega_{n}$, defined by Eq. (19), has been tabulated by Chandrasekhar (1932). The definition (24) of $\eta$ and the relation (25) between $\eta$ and $\alpha$ are consistent with the formulae derived in the case of an isothermal gas (see Chavanis 2002a). This connexion is particularly relevant if we interpret the constant $K$ as a polytropic temperature $\Theta_{\gamma}$ (see Chandrasekhar 1932, p. 86). For $n \rightarrow+\infty$, $\Theta_{\gamma}=T$ and the parameter $\eta$ reduces to the corresponding one for isothermal spheres $\left(\eta \sim \eta_{\infty} / n, \eta_{\infty}=\frac{\beta G M m}{R}\right.$, $\beta=1 / k T)$.

The function $\eta(\alpha)$ is represented in Fig. 5 for different values of the polytropic index $n$. Instead of $\alpha$, we could have used the density contrast

$\mathcal{R} \equiv \frac{\rho_{0}}{\rho(R)}=\theta(\alpha)^{-n}$,

which also provides a relevant parametrization of the solutions. Using the Lane-Emden Eq. (7) and the definition

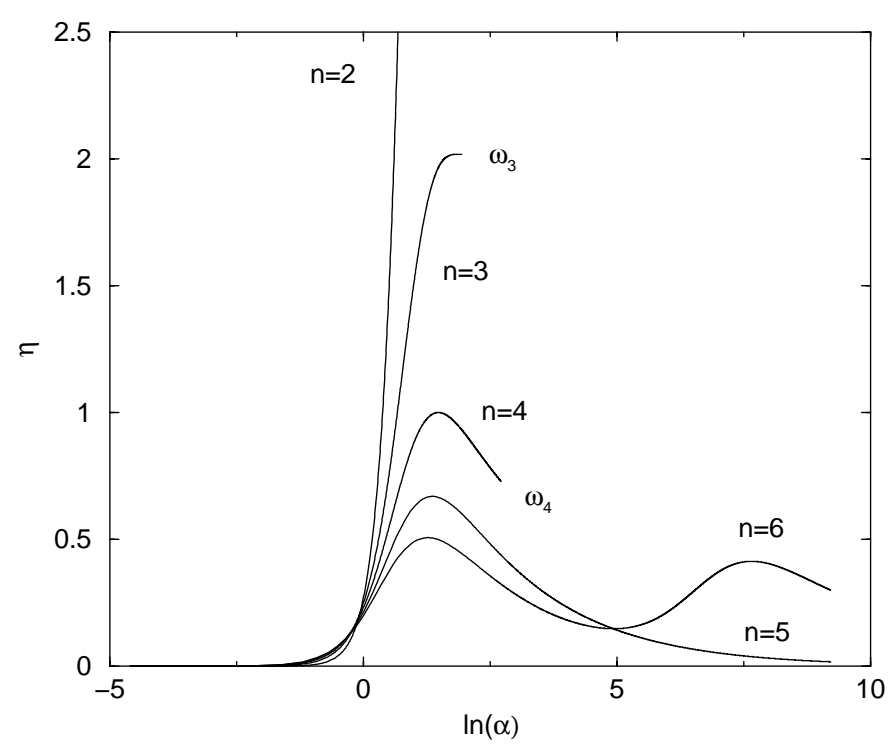

Fig. 5. Mass-density profiles for polytropic configurations with index $n=2,3,4,5,6$. A mass peak appears for the first time for the critical index $n=3$.

of the Milne variables (15), it is straightforward to check that the condition of extremum $\mathrm{d} \eta / \mathrm{d} \alpha=0$ is equivalent to

$u_{0}=\frac{n-3}{n-1} \equiv u_{\mathrm{s}}$

where, by definition, $u_{0}=u(\alpha)$ and $u_{\mathrm{s}}$ refers to the singular solution (9). For $n \rightarrow \infty$, we recover the condition $u_{0}=1$ previously derived for isothermal configurations (Chavanis 2002a). The values of $\alpha$ for which $\eta$ is extremum are given by the intersection(s) between the solution curve in the $(u, v)$ plane and the line $u=u_{s}$ (see Figs. 2-4). For $1<n<3, u_{\mathrm{s}}<0$ and there is no intersection. The mass-density relation is therefore monotonous. For $3 \leq n \leq 5$, the curve $\eta(\alpha)$ presents a single maximum at $\alpha_{1}$. For $n=3$, this maximum is reached at the extremity of the curve $\left(\alpha_{1}=\xi_{1}\right)$. For $n=5, \xi_{1} \rightarrow+\infty$ and the function $\eta(\alpha)$ is explicitly given by

$\eta=\frac{\alpha^{5 / 2}}{3\left(1+\frac{1}{3} \alpha^{2}\right)^{3 / 2}}$.

The maximum of $\eta$ is located at $\alpha_{1}=\sqrt{15}$. Finally, for $n>$ 5 , the mass-density relation presents an infinite number of damped oscillations since the line defined by Eq. (28) passes through the center of the spiral in the $(u, v)$ plane. If we denote by $\alpha_{k}$ the locii of the extrema of $\eta(\alpha)$, these values asymptotically follow the geometric progression

$\alpha_{k} \sim\left[\mathrm{e}^{\frac{2(n-1) \pi}{\sqrt{7 n^{2}-22 n-1}}}\right]^{k} \quad(k \rightarrow \infty$, integer $)$,

obtained by substituting the asymptotic expansion (12) in Eq. (28). For $n \rightarrow \infty$, we recover the ratio $\mathrm{e}^{2 \pi / \sqrt{7}}=$ $10.74 \ldots$ corresponding to classical isothermal configurations (Semelin et al. 1999; Chavanis 2002a). 
From the above results, it is clear that restricted polytropic spheres with index $n \geq 3$ can exist only for

$\eta \leq-\alpha_{1}^{\frac{n+1}{n-1}} \theta^{\prime}\left(\alpha_{1}\right) \equiv \eta_{\max }$

This implies in particular the existence of a limiting mass (for a given confining radius $R$ ) such that

$M \leq M_{\max }=4 \pi \eta_{\max }\left[\frac{K(1+n)}{4 \pi G}\right]^{\frac{n}{n-1}} R^{\frac{n-3}{n-1}}$.

For $n=\infty$, we recover the limiting mass $M_{\mathrm{c}}=2.52 \frac{R k T}{G m}$ above which an isothermal sphere cannot sustain selfgravity (see, e.g., Chavanis 2002b). Alternatively, for a given mass $M$ and radius $R$, the inequality (31) implies the existence of a minimum value of the polytropic temperature $\Theta_{\gamma}=K m / k$. Indeed, $\Theta_{\gamma}$ is restricted by the inequality

$\Theta_{\gamma} \geq \frac{4 \pi G m}{k(1+n)}\left(\frac{M}{4 \pi \eta_{\max }}\right)^{\frac{n-1}{n}} \frac{1}{R^{\frac{n-3}{n}}}$.

In the limit $n=\infty$, we recover the critical temperature $k T_{\mathrm{c}}=\frac{G M m}{2.52 R}$ below which an isothermal sphere is expected to collapse (Lynden-Bell \& Wood 1968). This critical point, corresponding to $\mathrm{d} \eta / \mathrm{d} \alpha=0$, appears for the first time for the index $n=3$. This observation will take a deeper physical significance in the stability analysis performed in the following section. For $n \leq 3$, the mass-density profile is monotonous and $\eta \leq \omega_{n}$. The total mass (resp. temperature) of confined polytropes is always smaller (resp. larger) than the corresponding one for isolated polytropes. However, this bound does not correspond to a condition of extremum $\mathrm{d} \eta / \mathrm{d} \alpha=0$ but rather to the impossibility of constructing polytropes with $\alpha>\xi_{1}$ (since $\theta$ can become negative).

\section{Dynamical stability of polytropic gas spheres}

\subsection{The equation of radial pulsations}

We shall now investigate the dynamical stability of polytropic gas spheres against radial perturbations. We use a method and presentation similar to that adopted for isothermal spheres in Chavanis (2002a). The equations describing the motions of a gaseous star are the equation of continuity, the Euler equation and the Poisson equation

$\frac{\partial \rho}{\partial t}+\nabla(\rho \boldsymbol{v})=0$

$\frac{\partial \boldsymbol{v}}{\partial t}+(\boldsymbol{v} \nabla) \boldsymbol{v}=-\frac{1}{\rho} \nabla p-\nabla \Phi$

$\Delta \Phi=4 \pi G \rho$.

It is possible to show that viscosity does not change the onset of instability. Therefore, for simplicity, we have directly used the Euler equation instead of the Navier-Stokes equation. We assume furthermore that the pressure and the density are related to each other by the polytropic equation of state (1). We write small perturbations around equilibrium in the form

$\boldsymbol{v}=\delta \boldsymbol{v}(\boldsymbol{r}, t), \quad \rho=\bar{\rho}+\delta \rho(\boldsymbol{r}, t)$,

$p=\bar{p}+\delta p(\boldsymbol{r}, t), \quad \Phi=\bar{\Phi}+\delta \Phi(\boldsymbol{r}, t)$,

where the bar refers to the stationary solution (in the following we shall drop the bar). The linearized equations for the perturbations are

$\rho \frac{\partial \delta \boldsymbol{v}}{\partial t}=-K \gamma \nabla\left(\rho^{\gamma-1} \delta \rho\right)-\delta \rho \nabla \Phi-\rho \nabla \delta \Phi$,

$\frac{\partial \delta \rho}{\partial t}+\nabla(\rho \delta \mathbf{v})=0$

$\Delta \delta \Phi=4 \pi G \delta \rho$.

Restricting ourselves to radial perturbations and writing the time dependance of the perturbation in the form $\delta v \sim$ $\mathrm{e}^{\lambda t}, \delta \rho \sim \mathrm{e}^{\lambda t}, \ldots$, the equations of the problem become

$\lambda \rho \delta v=-K \gamma \frac{\mathrm{d}}{\mathrm{d} r}\left(\rho^{\gamma-1} \delta \rho\right)-\delta \rho \frac{\mathrm{d} \Phi}{\mathrm{d} r}-\rho \frac{\mathrm{d} \delta \Phi}{\mathrm{d} r}$,

$\lambda \delta \rho+\frac{1}{r^{2}} \frac{\mathrm{d}}{\mathrm{d} r}\left(\rho r^{2} \delta v\right)=0$

$\frac{1}{r^{2}} \frac{\mathrm{d}}{\mathrm{d} r}\left(r^{2} \frac{\mathrm{d} \delta \Phi}{\mathrm{d} r}\right)=4 \pi G \delta \rho$.

We introduce the function $q(r)$ by the relation

$\delta \rho=\frac{1}{4 \pi r^{2}} \frac{\mathrm{d} q}{\mathrm{~d} r}$.

Physically, $q(r)$ represents the mass perturbation $\delta M(r)=$ $\int_{0}^{r} \delta \rho 4 \pi r^{2} \mathrm{~d} r$ within the sphere of radius $r$. Thus, by definition, $q(0)=0$. Then, it is readily seen that the Poisson equation (44) is equivalent to

$\frac{\mathrm{d} \delta \Phi}{\mathrm{d} r}=\frac{G q}{r^{2}}$,

which is just the perturbed Gauss theorem (4). On the other hand, the continuity Eq. (43) leads to the relation

$\delta v=-\frac{\lambda}{4 \pi \rho r^{2}} q$

Substituting these results back into Eq. (42), we obtain

$\frac{\lambda^{2}}{4 \pi r^{2}} q=K \gamma \frac{\mathrm{d}}{\mathrm{d} r}\left(\rho^{\gamma-1} \frac{1}{4 \pi r^{2}} \frac{\mathrm{d} q}{\mathrm{~d} r}\right)+\frac{1}{4 \pi r^{2}} \frac{\mathrm{d} q}{\mathrm{~d} r} \frac{\mathrm{d} \Phi}{\mathrm{d} r}+\frac{G \rho}{r^{2}} q$.

Using the condition of hydrostatic equilibrium (3), the foregoing equation can be rewritten

$K \gamma \frac{\mathrm{d}}{\mathrm{d} r}\left(\frac{\rho^{\gamma-2}}{4 \pi r^{2}} \frac{\mathrm{d} q}{\mathrm{~d} r}\right)+\frac{G q}{r^{2}}=\frac{\lambda^{2}}{4 \pi \rho r^{2}} q$,

which is the required equation of radial pulsations for a polytrope. 


\subsection{The condition of marginal stability}

Considering the case of marginal stability $\lambda=0$ and introducing the dimensionless variables defined in Sect. 2, we can reduce the equation of radial pulsations to the form

$\frac{\mathrm{d}}{\mathrm{d} \xi}\left(\frac{\theta^{1-n}}{\xi^{2}} \frac{\mathrm{d} F}{\mathrm{~d} \xi}\right)+\frac{n F}{\xi^{2}}=0$

with $F(0)=0$. Denoting by $\mathcal{L}$ the differential operator appearing in Eq. (50) and using the Lane-Emden Eq. (7), we easily establish that

$$
\begin{aligned}
\mathcal{L}\left(\xi^{2} \theta^{\prime}\right) & =\frac{\mathrm{d}}{\mathrm{d} \xi}\left(\frac{\theta^{1-n}}{\xi^{2}} \frac{\mathrm{d}}{\mathrm{d} \xi}\left(\xi^{2} \theta^{\prime}\right)\right)+n \theta^{\prime} \\
& =-\frac{\mathrm{d}}{\mathrm{d} \xi}\left(\theta^{1-n} \times \theta^{n}\right)+n \theta^{\prime}=(n-1) \theta^{\prime}, \\
\mathcal{L}\left(\xi^{3} \theta^{n}\right) & =\frac{\mathrm{d}}{\mathrm{d} \xi}\left(\frac{\theta^{1-n}}{\xi^{2}} \frac{\mathrm{d}}{\mathrm{d} \xi}\left(\xi^{3} \theta^{n}\right)\right)+n \xi \theta^{n} \\
& =(3+n) \theta^{\prime}+\xi n \theta^{\prime \prime}+n \xi \theta^{n}=(3-n) \theta^{\prime} .
\end{aligned}
$$

Therefore, the general solution of Eq. (50) is

$F(\xi)=c_{1}\left(\xi^{3} \theta^{n}+\frac{n-3}{n-1} \xi^{2} \theta^{\prime}\right)$,

where $c_{1}$ is an arbitrary constant. The connexion with isothermal configurations (see Chavanis 2002a) is particularly obvious if we make the correspondance $\theta^{\prime} \leftrightarrow \psi^{\prime}$ and $\theta^{n} \leftrightarrow \mathrm{e}^{-\psi}$.

\subsection{Boundary conditions}

The equation of pulsations (49) must be supplemented by appropriate boundary conditions. For self-confined polytropes $(1<n<5)$, we require that the Lagrangian derivative of the pressure vanishes at the surface of the configuration, i.e.

$\frac{\mathrm{d}}{\mathrm{d} t} \delta p+\delta v \frac{\mathrm{d} p}{\mathrm{~d} r}=0, \quad$ at $r=R$.

Using Eqs. (45), (47), this condition can be rewritten

$\frac{p}{\rho}\left(\frac{\mathrm{d} q}{\mathrm{~d} r}-\frac{q}{\rho} \frac{\mathrm{d} \rho}{\mathrm{d} r}\right)=0, \quad$ at $r=R$,

or, in dimensionless form,

$\theta \frac{\mathrm{d} F}{\mathrm{~d} \xi}-n \frac{\mathrm{d} \theta}{\mathrm{d} \xi} F=0, \quad$ at $\xi=\xi_{1}$.

The index of the marginally stable polytrope is obtained by substituting the solution (53) in the boundary condition (56). Since $\theta\left(\xi_{1}\right)=0$, this yields $n=3$. Therefore, the transition from stability to instability occurs for a polytropic index $n=3$ or, equivalently, for an adiabatic index $\gamma=4 / 3$. Of course, this result is well-known and could have been obtained from the general theorems of stellar pulsation (see, e.g., Cox 1980). However, we provide here an alternative method based on the explicit resolution of the equation of pulsations for polytropes.

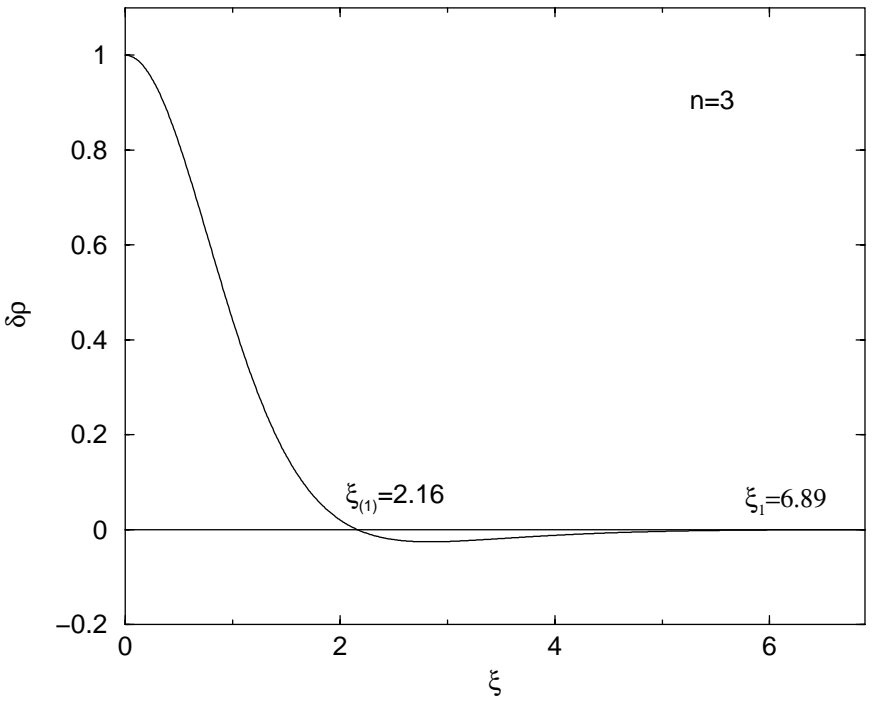

Fig. 6. Density perturbation profile for the critical polytrope of index $n=3$.

According to Eq. (45), the perturbation profile at the point of marginal stability is given by

$\frac{\delta \rho}{\rho_{0}}=\frac{1}{4 \pi \xi^{2}} \frac{\mathrm{d} F}{\mathrm{~d} \xi}$,

with the expression (53) for $F(\xi)$. Simplifying the derivative with the aid of the Lane-Emden Eq. (7), we obtain

$\frac{\delta \rho}{\rho}=\frac{3}{4 \pi} c_{1}\left(\frac{2}{n-1}-v\right)$,

where $v$ is the Milne variable (15). For $n=3$, it reduces to

$\delta \rho=\frac{3}{4 \pi} c_{1} \rho_{0} \theta^{3}(1-v)$.

This perturbation profile is plotted in Fig. 6. It has one node at $\xi_{(1)}=2.16 \ldots$ On the other hand, the velocity profile is given by Eq. (47). Normalizing with a typical velocity $c_{\mathrm{S}}$, we find that

$\frac{\delta v}{c_{\mathrm{S}}}=-\frac{\lambda^{\prime}}{4 \pi} c_{1} \frac{\theta^{\prime}}{\theta^{n}}\left(\frac{n-3}{n-1}-u\right)$.

We assume that we are just at the onset of the instability $\left(\lambda^{\prime}=0^{+}\right)$so that Eq. (60) is applicable with $\lambda^{\prime}>0$. Substituting explicitly for $n=3$, we get

$\frac{\delta v}{c_{\mathrm{S}}}=-\frac{\lambda^{\prime}}{4 \pi} c_{1} \xi$

and we observe that the velocity is proportional to the radial distance (see also Cox 1980).

For polytropes confined within a box, the boundary condition consistent with the conservation of mass is $F(\alpha)=0$. With the expression (53) for $F(\xi)$, this yields

$u_{0}=\frac{n-3}{n-1}$

which is precisely Eq. (28). Therefore, the point of marginal stability coincides with the point of maximum 


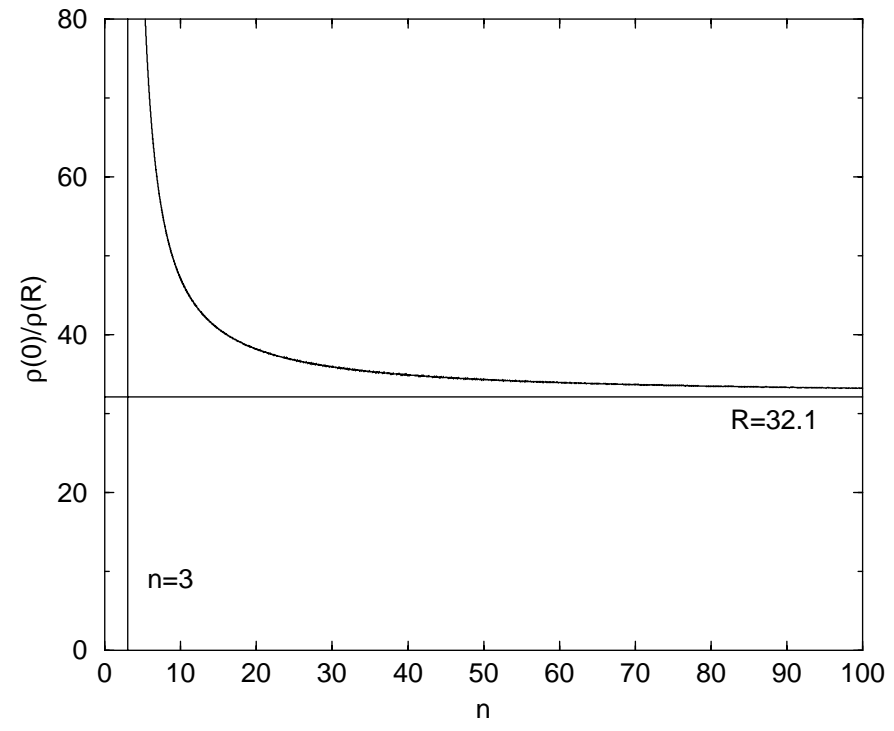

Fig. 7. Critical density contrast as a function of the polytropic index $n$.

mass (or minimum temperature) in the series of equilibrium. For $n<3$, restricted polytropes are always stable since the function $\eta(\alpha)$ is monotonous. For $3 \leq n \leq 5$, the configurations with $\alpha>\alpha_{1}$ are unstable but there are no secondary instabilities. For $n>5$, a first mode of stability is lost for $\alpha=\alpha_{1}$. Subsequent oscillations in the mass-density profile are associated with secondary instabilities. A summary of the stability limits for polytropic gas spheres is given in Table 1 . In Fig. 7 we have plotted the density contrast at the critical point $\alpha=\alpha_{1}$ as a function of the index of the polytrope. For $n \leq 3$, the polytropes are stable and $\mathcal{R}_{\mathrm{c}}=+\infty$. For $n>3$, only configurations with a density contrast $\mathcal{R} \leq \mathcal{R}_{\mathrm{c}}$ are stable. For $n \rightarrow+\infty$, the critical density contrast tends to its classical value $\mathcal{R}_{\mathrm{c}}=32.1$ (see Chavanis 2002a).

Using Eqs. (58), (60), the profiles of density and velocity that trigger the instabilities at the critical points can be written

$\frac{\delta \rho}{\rho}=\frac{3}{4 \pi} c_{1}\left(v_{\mathrm{s}}-v\right)$

$\frac{\delta v}{c_{\mathrm{S}}}=-\frac{\lambda^{\prime}}{4 \pi} c_{1} \frac{\theta^{\prime}}{\theta^{n}}\left(u_{\mathrm{s}}-u\right)$.

where we recall that $\xi \leq \alpha_{k}$ for the mode of instability of order $k$. We can determine the qualitative behavior of these profiles graphically by considering the intersections between the solution curve in the $(u, v)$ plane and the lines $v=v_{\mathrm{s}}$ and $u=u_{\mathrm{s}}$ (see Figs. 2-4). These intersections determine the nodes of the perturbation profile. For $3 \leq n \leq 5$, there is only one intersection since the $(u, v)$ curve is monotonous. For $n=5$, we obtain the analytical solutions

$\frac{\delta \rho}{\rho}=\frac{3}{8 \pi} c_{1} \frac{3-\xi^{2}}{3+\xi^{2}}$

$\frac{\delta v}{c_{\mathrm{S}}}=\frac{\lambda^{\prime}}{72 \pi} c_{1} \xi\left(\xi^{2}-15\right)$.
Table 1. Summary of the stability limits for polytropic gas spheres.

\begin{tabular}{lll}
\hline \hline index & unconfined & box - confined \\
\hline$n=1$ & stable & stable \\
$n=3$ & unstable & unstable for $\alpha>\alpha_{1} ;$ \\
& & no secondary instability \\
$n=5$ & infinite & unstable for $\alpha>\alpha_{1} ;$ \\
& mass & $\begin{array}{l}\text { secondary instabilities at } \\
\alpha_{2}, \alpha_{3}, \ldots\end{array}$ \\
& & \\
$n=\infty$ & &
\end{tabular}

The perturbation $\delta \rho$ has its node at $\xi_{(1)}=\sqrt{3}$. For $n>5$, there are several intersections with the spiral since the lines $v=v_{\mathrm{s}}$ and $u=u_{\mathrm{s}}$ pass through the fixed point. The description of the perturbation profiles is similar to the one given for isothermal spheres $(n=\infty)$. The density profile of the fundamental mode of instability (at $\alpha=\alpha_{1}$ ) has only one node. High order modes of instability (at $\alpha=\alpha_{k}, k>1$ ) present numerous oscillations whose nodes asymptotically follow a geometric progression. We refer to our previous paper (Chavanis 2002a) for a more precise description of these results.

\section{Generalized thermodynamics and Tsallis entropy}

Recently, Taruya \& Sakagami (2002) have investigated the stability of polytropic spheres within the framework of generalized thermodynamics. It has been argued by Tsallis and co-workers that ordinary statistical mechanics and thermodynamics does not describe correctly systems with long-range interactions, which are in essence non extensive. A family of functionals of the form

$S_{q}=-\frac{1}{q-1} \int\left(f^{q}-f\right) \mathrm{d}^{3} \boldsymbol{r} \mathrm{d}^{3} \boldsymbol{v}$,

known as Tsallis entropies, has been introduced to extend the classical Boltzmann-Gibbs statistical mechanics to these systems. These functionals are labeled by a parameter $q$. The Boltzmann entropy is recovered in the limit $q \rightarrow 1$. This new formalism has been applied in various domains of physics, astrophysics, fluid mechanics, biology, economy etc. and it extends the results obtained with ordinary statistical mechanics. In the case of self-gravitating systems, it was shown by Plastino \& Plastino (1993) that the extremalization of the Tsallis entropy at fixed mass and energy yields a polytropic equation of state of the form (1). The parameter $q$ is related to the index $n$ of the polytrope by the relation

$n=\frac{1}{q-1}+\frac{3}{2}, \quad(q \geq 1)$. 
Then, Taruya \& Sakagami (2002) investigated the stability problem in the microcanonical ensemble by extending the analysis of Padmanabhan (1989) for the classical Antonov instability. Polytropic configurations are said to be stable if they correspond to (generalized) entropy max- $^{-}$ ima at fixed mass $M$ and energy $E$. Taruya \& Sakagami showed that, for $n>5$, an equilibrium exists only above a critical energy $E_{\mathrm{c}}$ depending on the index of the configuration. In the limit $n \rightarrow+\infty$, the Antonov result $E_{\mathrm{c}}=-0.335 G M^{2} / R$ obtained with the Boltzmann entropy is recovered. Furthermore, they showed that the onset of instability coincides with the point of minimum energy in the series of equilibrium in agreement with standard turning point analysis (e.g., Katz 1978). For $n<5$, there is no critical value of energy and the polytropic configurations are stable. These results differ from those obtained in the present paper where it is found that the index marking the transition from stability to instability is $n=3$ (in agreement with standard theorems of stellar pulsations). The origin of this discrepency is certainly related to the inequivalence of statistical ensembles in extended thermodynamics, like in ordinary thermodynamics, for self-gravitating systems (see, e.g., Padmanabhan 1990; Chavanis 2002c; Chavanis et al. 2002). Taruya \& Sakagami work in the microcanonical ensemble and maximize Tsallis entropy at fixed mass and energy. On the contrary, in our study, we keep the polytropic temperature $\Theta_{\gamma}=K m / k$ constant, which corresponds to the canonical description. It would be of interest to study the maximization of Tsallis free energy at fixed mass and temperature to see if it provides the same conditions of stability as our dynamical approach based on the Navier-Stokes equations. For the Boltzmann entropy, a clear connexion was found between dynamical stability and thermodynamical stability in the canonical ensemble (Semelin et al. 2001; Chavanis 2002a) and it is desirable to check whether this connexion is preserved by Tsallis generalized thermodynamics. This requires in particular to define properly the notion of temperature and free energy in the generalized sense.

In Figs. 8-11, we have represented the equilibrium phase diagram (for different values of $n$ ) obtained in the framework of extended thermodynamics, interpreting the parameter $\eta$ as a generalized inverse temperature. The temperature-energy curve is defined in a parametric form by the equations

$\eta=-\alpha^{\frac{n+1}{n-1}} \theta^{\prime}(\alpha)$

$\Lambda \equiv-\frac{E R}{G M^{2}}=-\frac{1}{n-5}\left[\frac{3}{2}\left(1-\frac{1}{v(\alpha)}\right)+\frac{n-2}{n+1} \frac{u(\alpha)}{v(\alpha)}\right]$.

The expression (70) for the energy has been derived by Taruya \& Sakagami (2002). For $n<3$, there is no turning point in the diagram, so the polytropes are always stable. For $3<n<5$, the inverse temperature $\eta$ presents a maximum but not the energy. Therefore, the polytropes are always stable in the microcanonical ensemble but they are unstable in the canonical ensemble after the turning

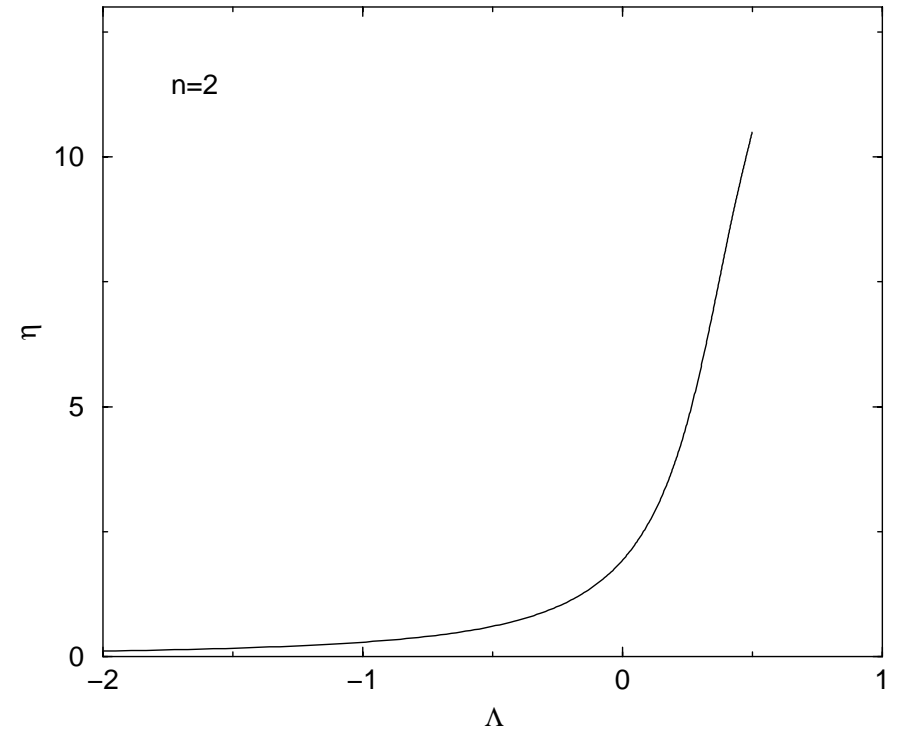

Fig. 8. Phase diagram for $n=2$.

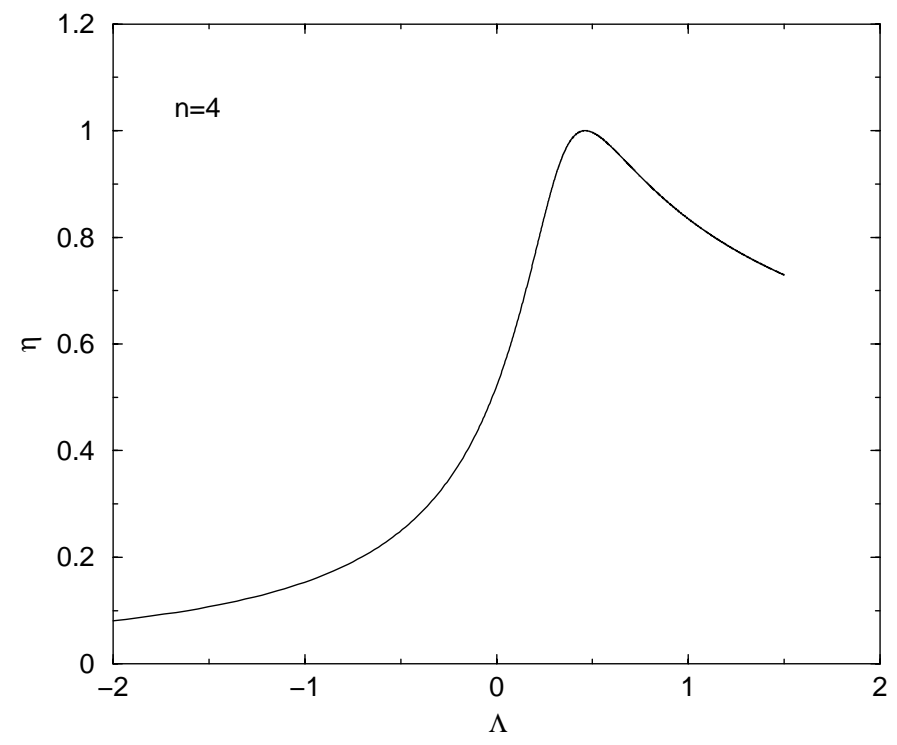

Fig. 9. Phase diagram for $n=4$.

point. It can be noted that the unstable region has a negative specific heat $C \sim \frac{\mathrm{d} \Lambda}{\mathrm{d} \eta}<0$ (in the generalized sense). For $n>5$, the temperature and the energy both present an infinite number of extrema. For $n \sim 6$, there are crossing points at which two solutions have the same value of temperature and energy but a different density contrast (to our knowledge, this is the first time such a situation is reported). The polytropes are unstable in the canonical ensemble after the first turning point of temperature and they are unstable in the microcanonical ensemble after the first turning point of energy. These results are strikingly similar to those obtained with the classical Boltzmann entropy $(n=\infty)$.

It is interesting that the results obtained for isothermal gas spheres described by ordinary thermodynamics (Boltzmann entropy) can be extended to polytropic configurations by introducing a generalized functional (Tsallis 


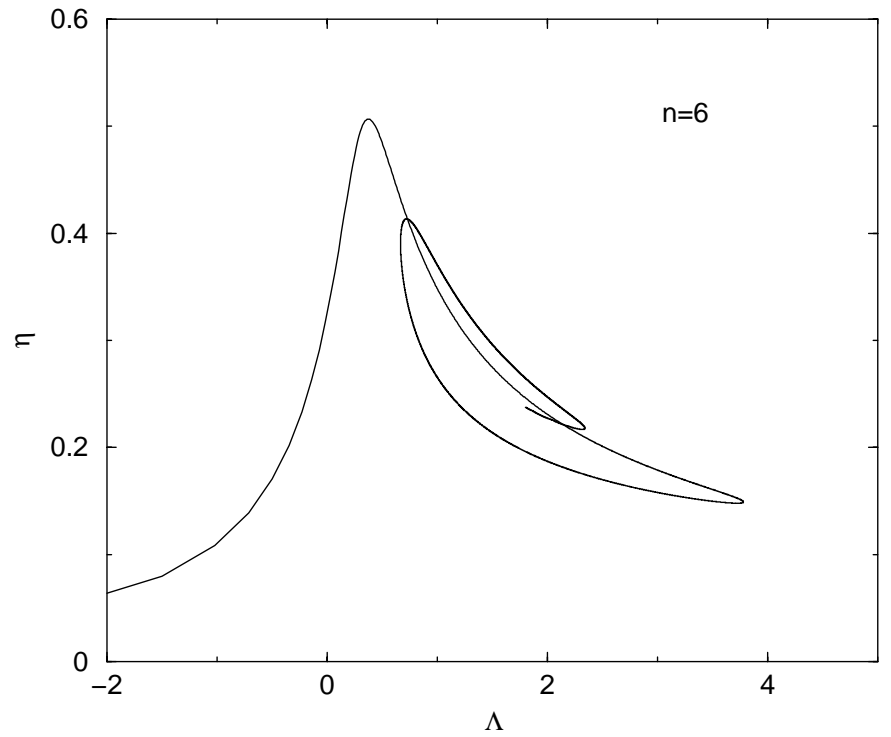

Fig. 10. Phase diagram for $n=6$.

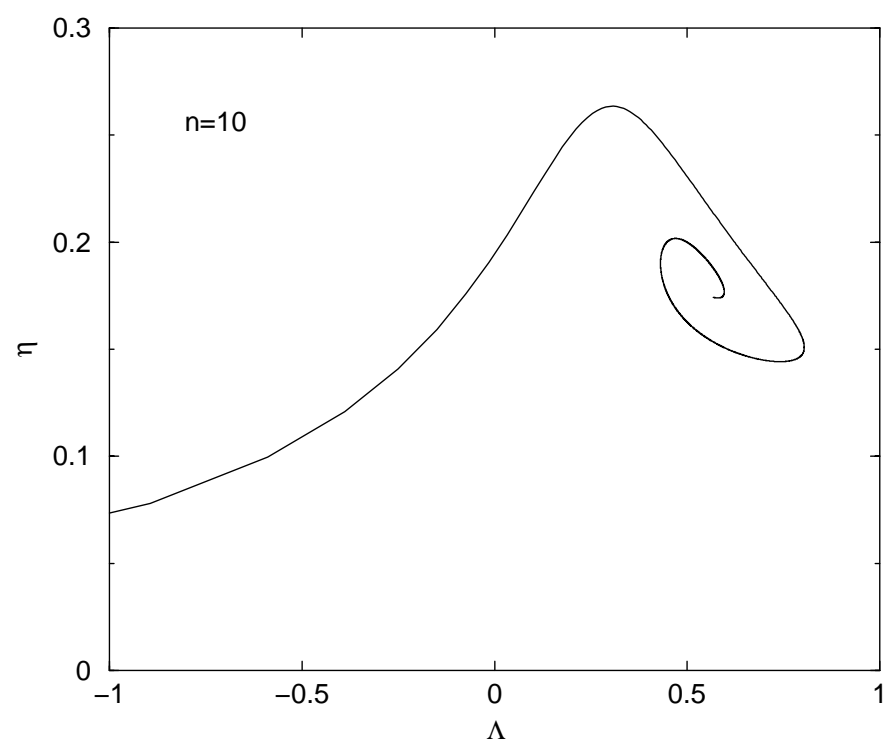

Fig. 11. Phase diagram for $n=10$.

entropy). This makes this new formalism of interest. However, we would like to make two comments. (i) First of all, Tsallis entropy does not describe polytropic gaseous stars although it yields an equation of state of the polytropic form. Indeed, Tsallis entropy predicts in addition a distribution function of the form

$f(\boldsymbol{r}, \boldsymbol{v})=A\left[\Phi_{0}-\Phi(\boldsymbol{r})-\frac{1}{2} v^{2}\right]^{1 /(q-1)}$,

whereas in polytropic stars, the distribution function corresponds to a local thermodynamical equilibrium (L.T.E) characterized by a Maxwellian distribution of velocities

$f(\boldsymbol{r}, \boldsymbol{v})=\left(\frac{m}{2 \pi k T(\boldsymbol{r})}\right)^{3 / 2} \rho(\boldsymbol{r}) \mathrm{e}^{-\frac{m v^{2}}{2 k T(\boldsymbol{r})}}$,

with a local temperature $T(\boldsymbol{r})$ (recall that, for convective equilibrium, the entropy is uniform not the temperature). In view of these remarks, the connexion between generalized thermodynamical stability (in the canonical ensemble) and dynamical stability with respect to NavierStokes equations is not obvious. It is however expected to be true since the condition of dynamical stability is consistent with the turning point argument of thermodynamical stability. (ii) Tsallis entropy could describe a particular class of stellar systems, apparently not observed in nature, known as stellar polytropes (see Binney \& Tremaine 1987). These systems have the same structure as polytropic gas spheres in physical space but not in phase space (the equivalence only occurs for a polytropic index $n=\infty$, i.e. for an isothermal configuration). This distinction is important because isolated stellar polytropes are always stable with respect to the Vlasov (or collisionless Boltzmann) equation (see Binney \& Tremaine 1987) while isolated polytropic stars are unstable with respect to the Navier-Stokes equations for $n \geq 3$. Generalized thermodynamics could be developed (at least formally) for this particular class of stellar systems.

However, generalized thermodynamics is often presented as a way of solving the "problems" associated with the use of the Boltzmann entropy in systems with longrange interactions and we would like to criticize this interpretation. For self-gravitating systems, the "problems" usually reported are the absence of global entropy maximum (resulting in the "infinite mass problem" and the "gravothermal catastrophe"), the negative specific heats, the inequivalence of statistical ensembles and the absence of a thermodynamical limit. It should be noted that the Tsallis entropy displays the same phenomena, at least for values of $q<9 / 7$ (i.e. $n>5$ ) considered by Taruya \& Sakagami (2002). On the other hand, these relatively unusual phenomena should not cause surprise if one recognizes that self-gravitating systems are relatively exceptional among $N$-body systems. For example the "infinite mass problem" (see, e.g., Binney \& Tremaine 1987) is just a mathematical curiosity. There is no justification in maximizing entropy in an infinite domain even if an entropy maximum exists. Due to kinetic effects, the relaxation is incomplete and the ergodic hypothesis which sustains the statistical analysis is necessarily restricted to a finite region of space. This incomplete relaxation is qualitatively discussed by Lynden-Bell (1967) in his statistical description of the "violent relaxation" of collisionless stellar systems (e.g., elliptical galaxies). A similar limitation occurs in the context of two-dimensional turbulence to understand the confinement of vortices that form after a rapid merging (see, e.g., Chavanis \& Sommeria 1998 and in particular Brands et al. 1999 for a discussion of Tsallis entropy in fluid dynamics). This incomplete relaxation can be described by kinetic equations of the form proposed by Chavanis et al. (1996) with a space dependant diffusion coefficient. Convincing numerical evidence of this kinetic confinement has been given by Robert \& Rosier (1997) in two-dimensional turbulence. In addition, in astrophysics, specific processes (e.g., the evaporation of stars, tidal effects, etc.) must be taken into account and can restrict the domain of applicability of statistical 
mechanics. These limitations are usually accounted for by introducing truncated models like the Michie-King model for globular clusters or the models proposed by Stiavelli \& Bertin (1987) or Hjorth \& Madsen (1993) for elliptical galaxies. These models lead to composite configurations with an isothermal core and a polytropic envelope. With these modifications (justified by precise physical arguments), ordinary statistical mechanics provides in general a good explanation of astrophysical phenomena and is consistent with observations. Note that the above-mentioned truncated models differ from pure polytropes, so that they cannot be justified by generalized thermodynamics ${ }^{1}$. On the other hand, the "gravothermal catastrophe" (LyndenBell \& Wood 1968) should not throw doubt on the validity of statistical mechanical arguments applied to selfgravitating systems. On the contrary, it is gratifying that standard thermodynamics is consistent with the natural tendency of self-gravitating systems to undergo a gravitational collapse (Jeans instability). The gravothermal catastrophe has been confirmed by sophisticated numerical simulations (see, e.g., Larson 1970; Cohn 1980) based on standard kinetic theory (Landau-Fokker-Planck equations) and it is expected to be at work in globular clusters. The existence of negative specific heats in the microcanonical ensemble (and the resulting inequivalence of statistical ensembles) is a direct consequence of the Virial theorem for self-gravitating systems and is not in contradiction with ordinary thermodynamics (Lynden-Bell \& LyndenBell 1977; Padmanabhan 1990). Finally, self-gravitating systems have a well-defined (albeit unusual) thermodynamical limit in which the number of particles $N$ and the volume $R^{3}$ go to infinity keeping $N / R$ fixed (see, e.g., de Vega \& Sanchez 2001).

\footnotetext{
1 Recently, Fa \& Pedron (2001) have proposed to describe elliptical galaxies by an extended King model using Tsallis entropy. They obtained a good fit for NGC 3379. However, we believe that this agreement is fortuitous. An even better fit has been obtained by Hjorth \& Madsen (1993) with a different model justified by more convincing physical arguments. Their model has an isothermal (instead of a polytropic) inner region in agreement with Lynden-Bell's statistical mechanics of violent relaxation. The polytropic envelope is justified by incomplete relaxation (or equivalently by a lack of ergodicity) when we depart from the central mixing region. Their model differs from the Michie-King model because the system is collisionless and not subject to tidal forces (King's models, which can be derived rigorously from the Fokker-Planck equation, only apply to tidally truncated collisional stellar systems such as globular clusters). It is seen in Fig. 3 of Fa \& Pedron (2001) that an isothermal distribution in the inner region provides a sensibly better fit to observations than a polytropic one (it can reproduce the oscillations). In addition, the model of Hjorth \& Madsen (1993) has only one fitting parameter $\Phi_{0}$ (the central potential) instead of two $\Phi_{0}$ and $q$ in Fa \& Pedron's analysis. These remarks tend to show that generalized thermodynamics is not necessary to understand the structure of self-gravitating systems. A similar conclusion has been reached by Brands et al. (1999) in two-dimensional turbulence.
}

Therefore, we are tempted to believe that ordinary statistical mechanics is still relevant to describe selfgravitating systems (and two-dimensional vortices). An isothermal distribution corresponds to the most probable distribution reached by a system after a complex evolution during which microscopic information is lost. Accordingly, it maximizes the Boltzmann entropy, which is a measure of the number of microstates associated with a given macrostate (see, e.g., Ogorodnikov 1965; LyndenBell 1967). This definition of the entropy does not depend whether the particles are in interaction or not. The effects of non-locality and non-extensivity intervene only when the entropy is maximized at fixed energy. It is implicitly assumed, however, that all accessible microstates are equiprobable, which is the fundamental postulate of statistical mechanics, and that the system converges at equilibrium towards the most probable (macroscopic) distribution. Generalized thermodynamics is interesting to extend standard results of thermodynamics to a larger class of functionals which possess nice mathematical properties, but it should not be presented (in our point of view) as an improvement or a replacement of classical thermodynamics.

We agree with Tsallis and co-workers that the kinetic theory of self-gravitating systems (see, e.g., Kandrup 1981) and two-dimensional vortices (see, e.g., Chavanis 2001) encounters some problems due to the occurence of memory effects, spatial delocalization and logarithmic divergences in the diffusion coefficient. For these reasons, and also for the problems of incomplete relaxation (lack of ergodicity) mentioned previously, the system may not necessarily reach the maximum entropy state described by the Boltzmann distribution, even in the mixing region. The deviation from the Boltzmann distribution is never too severe (see, e.g., the isothermal core of globular clusters and of elliptical galaxies) so that ordinary statistical mechanics provides a fairly good prediction from any initial condition. It may happen that the effective equilibrium distribution can be fitted by the $q$-distribution proposed by Tsallis and co-workers better than by the Boltzmann distribution. This is the case for some examples of statistical equilibrium in two-dimensional turbulence (see the discussion of Brands et al. 1999). However, this agreement may be fortuitous rather than dictated by a general physical principle since there is a parameter $q$ in the theory which, in practice, must be adjusted in each case. The fit can only improve since this family of distribution includes the Boltzmann distribution as a particular limit $(q=1)$. In fact, it is advocated by Tsallis (private communication) that the $q$-parameter is not free but uniquely determined by the microscopic dynamics of the system. It can be relatively easy to determine if the system is simple enough but it can also be very hard to determine in the case of complex systems like those involved in fluid mechanics and stellar dynamics. In that case, it must be regarded as a fitting parameter. Unfortunately, the power of prediction of the Tsallis theory is limited as long as a precise prescription for determining $q$ is not given. Tsallis entropy 
could be considered, however, as a heuristic attempt to take into account non-ergodic effects in complex systems. In view of these remarks, it would be of interest to see if the non Markovian and non-local generalized kinetic equations proposed by Kandrup (1981) in stellar dynamics and by Chavanis (2001) in vortex dynamics allow for a stationary distribution of the form conjectured by Tsallis, instead of the Boltzmann distribution. From our point of view, we believe that they do not select any "universal" distribution except in the approximation where (i) memory effects are ignored (ii) a local approximation is made (in the stellar context) (iii) ergodicity is assumed, in which case the classical Boltzmann distribution is obtained. It is hard to believe that extremely complicated effects of non ergodicity, spatial delocalization and memory can be encapsulated in a simple functional with a prescribed value of $q$. However, if it can be proved that these generalized kinetic equations rigorously converge towards a Tsallis distribution with $q \neq 1$, this would of course be a strong argument in favour of generalized thermodynamics. Unfortunately, the study of these generalized kinetic equations seems of considerable difficulty and will demand an extended effort.

\section{Conclusion}

This study has revealed that the striking results obtained by Antonov (1962) and Lynden-Bell \& Wood (1968) in their investigations on the thermodynamics of selfgravitating systems are not restricted to isothermal configurations but also occur for polytropic configurations. It is possible that this formal analogy is the mark of a generalized thermodynamics (Tsallis entropy) but this point needs to be discussed in greater detail. In any case, the study presented in this paper and in our companion papers (Chavanis 2002a,b) provide a unified description of the structure and stability of isothermal and polytropic gas spheres. This is a useful complement to the existing literature on the subject (Chandrasekhar 1932). Quite remarkably, the stability analysis can be performed analytically or by using graphical constructions. Unfortunately, our study is limited in its applications by the requirement of a confining box in the case of isothermal configurations and polytropes of index $n \geq 5$. In future works (in preparation), we shall extend our analytical results to the case of composite configurations made of an isothermal core and a polytropic envelope with index $n<5$. This is an important generalization for a better description of self-gravitating systems from the viewpoint of statistical mechanics and thermodynamics.

Acknowledgements. I acknowledge interesting comments from C. Tsallis and J. Perez on the first draft of this paper.
This work was initiated during my stay at the Institute for Theoretical Physics, Santa Barbara, during the program on Hydrodynamical and Astrophysical Turbulence (FebruaryJune 2000). This research was supported in part by the National Science Foundation under Grant No. PHY94-07194.

\section{References}

Antonov, V. A. 1962, Vest. Leningr. Gos. Univ., 7, 135

Binney, J., \& Tremaine, S. 1987, Galactic Dynamics (Princeton Series in Astrophysics)

Brands, H., Chavanis, P. H., Pasmanter, R., \& Sommeria, J. 1999, Phys. Fluids, 11, 3465

Chandrasekhar, S. 1931, ApJ, 74, 81

Chandrasekhar, S. 1942, An Introduction to the Theory of Stellar Structure (Dover)

Chavanis, P. H. 2001, Phys. Rev. E, 64, 026309

Chavanis, P. H. 2002a, A\&A, 381, 340

Chavanis, P. H. 2002b, A\&A, 381, 709

Chavanis, P. H. 2002c, to appear in Phys. Rev. E [cond-mat/0109294]

Chavanis, P. H., \& Sommeria, J. 1998, J. Fluid Mech., 356, 259

Chavanis, P. H., Sommeria, J., \& Robert, R. 1996, ApJ, 471, 385

Chavanis, P. H., Rosier, C., \& Sire, C. 2002, to appear in Phys. Rev. E [cond-mat/0107345]

Cohn, H. 1980, ApJ 242, 765

Cox, J. P. 1980, Theory of stellar pulsation (Princeton Series in Astrophysics)

de Vega, H. J., \& Sanchez, N. 2001 [astro-ph/0101568]

Fa, K. S., \& Pedron, I. T. 2001 [astro-ph/0108370]

Fowler, R. H. 1926, MNRAS, 87, 114

Hjorth, J., \& Madsen, J. 1993, MNRAS, 265, 237

Kandrup, H. 1981, ApJ, 244, 316

Katz, J. 1978, MNRAS, 183, 765

Larson, R. B. 1970, MNRAS, 147, 323

Lynden-Bell, D. 1967, MNRAS, 136, 101

Lynden-Bell, D., \& Lynden-Bell R. M. 1977, MNRAS, 181, 405

Lynden-Bell, D., \& Wood, R. 1968, MNRAS, 138, 495

Ogorodnikov, K. F. 1965, Dynamics of stellar systems (Pergamon)

Padmanabhan, T. 1989, ApJS, 71, 651

Padmanabhan, T. 1990, Phys. Rep., 188, 285

Plastino, A. R., \& Plastino, A. 1993, Phys. Lett. A, 174, 384

Robert, R., \& Rosier, C. 1997, J. Stat. Phys., 86, 481

Semelin, B., de Vega, H. J., Sanchez, N., \& Combes, F. 1999, Phys. Rev. D, 59, 125021

Semelin, B., Sanchez, N., \& de Vega, H.J. 2001, Phys. Rev. D, 63, 084005

Stiavelli, M., \& Bertin, G. 1987, MNRAS, 229, 61

Taruya, A., \& Sakagami, M. 2002, to appear in Physica A [cond-mat/0107494] 